\title{
A high-data-transfer-rate VME system for TCP-IP remote real-time control of the ITER in-vessel vision system
}

\author{
Rita Pereira ${ }^{a, *}$, Nuno Cruz ${ }^{\text {a }}$, Carlo Neri ${ }^{c}$, Carlos Correia ${ }^{\text {a,b }}$, \\ Carlos Varandas ${ }^{\text {a }}$ \\ a Associação EURATOM/IST, Centro de Fusão Nuclear, Instituto Superior Técnico, 1049-001 Lisbon, Portugal \\ ${ }^{\mathrm{b}}$ Grupo de Electrónica e Instrumentação, Departamento de Física, Universidade de Coimbra, 3004-516 Coimbra, Portugal \\ ${ }^{c}$ Associazione EURATOM-ENEA sulla Fusione, Centro Ricerche Frascati, C.P. 65, 00044 Frascati, Rome, Italy
}

\begin{abstract}
This paper describes the control and data acquisition system (CADAS) for the in-vessel vision system (IVVS) for ITER, which imposes special design requirements due to its reactor characteristics. CADAS provides remote real-time control of the motors (by TCP-IP), fast data acquisition (20 bytes/pixel at $100 \mathrm{kpixels} / \mathrm{s}$ ), error management and sustainable network data transfer rates (by TCP-IP). It is composed of a supervisory workstation connected by a 100 Mbit/s Ethernet channel to a remote VME crate, housing a board based on a PowerPC604R CPU running LynxOS, and two on-site developed intelligent modules. The architecture of these VME modules as well as the operation software and the application protocol are described. The test results are presented as well as an image obtained during the test procedures performed at the ENEA-Frascati Laboratory. (C) 2002 Elsevier Science B.V. All rights reserved.
\end{abstract}

Keywords: Data acquisition; Control; Remote control

\section{Introduction}

Any large fusion machine, especially those with Deuterium-Tritium (D-T) operation, such as International Thermonuclear Experimental Reactor (ITER), needs periodic in-vessel wall inspections to check for damage due to plasma disruptions. In addition, due to its size and to the large in-vessel activation, ITER requires the extensive use of both remote maintenance and high-resolution

\footnotetext{
* Corresponding author. Tel.: + 351-239-410-108; fax: + 351-239-829-158.

E-mail address: ritacp@lei.fis.uc.pt (R. Pereira).
}

imaging systems. These systems are the ITERequivalent of the JET in-vessel inspection system (IVIS), which is based on TV camera probes. These components have limited neutron radiation tolerance $\left(>10^{18}\right.$ neutrons $\left./ \mathrm{m}^{2}\right)$ and need to be removed during $\mathrm{D}-\mathrm{T}$ operation.

For this purpose a remotely controlled imaging system, based on scanning techniques of amplitude modulated (AM) laser beam, has been developed, following the ITER requirements. This complex in-vessel viewing system (IVVS) can be described as an AM laser beam deflected by a prism rotating around two orthogonal axes (tilt and pan), in such a way as to scan the in-vessel surface in a quasi-spherical viewing field. The 
difference between the amplitudes and phases of the reflected and incident beams are detected and computed aiming at constructing an image in which each pixel, in addition to intensity, carries depth information. From the amplitude signal a high-resolution picture of the target is obtained, while the phase shifting allows for target ranging. The final result is an image of the target with a resolution of $1 \mathrm{~mm}$ at $10 \mathrm{~m}$ viewing distance. The scanning of the in-vessel ITER surface is remotely controlled. Since part of the system (optical and mechanical IVVS apparatus [1]) operates in adverse conditions [2], an alarm system must also be foreseen. A dedicated computerised system controls all the system functionality and acquires all the analogue and digital information produced during the scanning process $(20$ bytes per pixel acquired every $10 \mu \mathrm{s})$. This information is then processed and displayed as images. Fig. 1 shows a block diagram of IVVS.
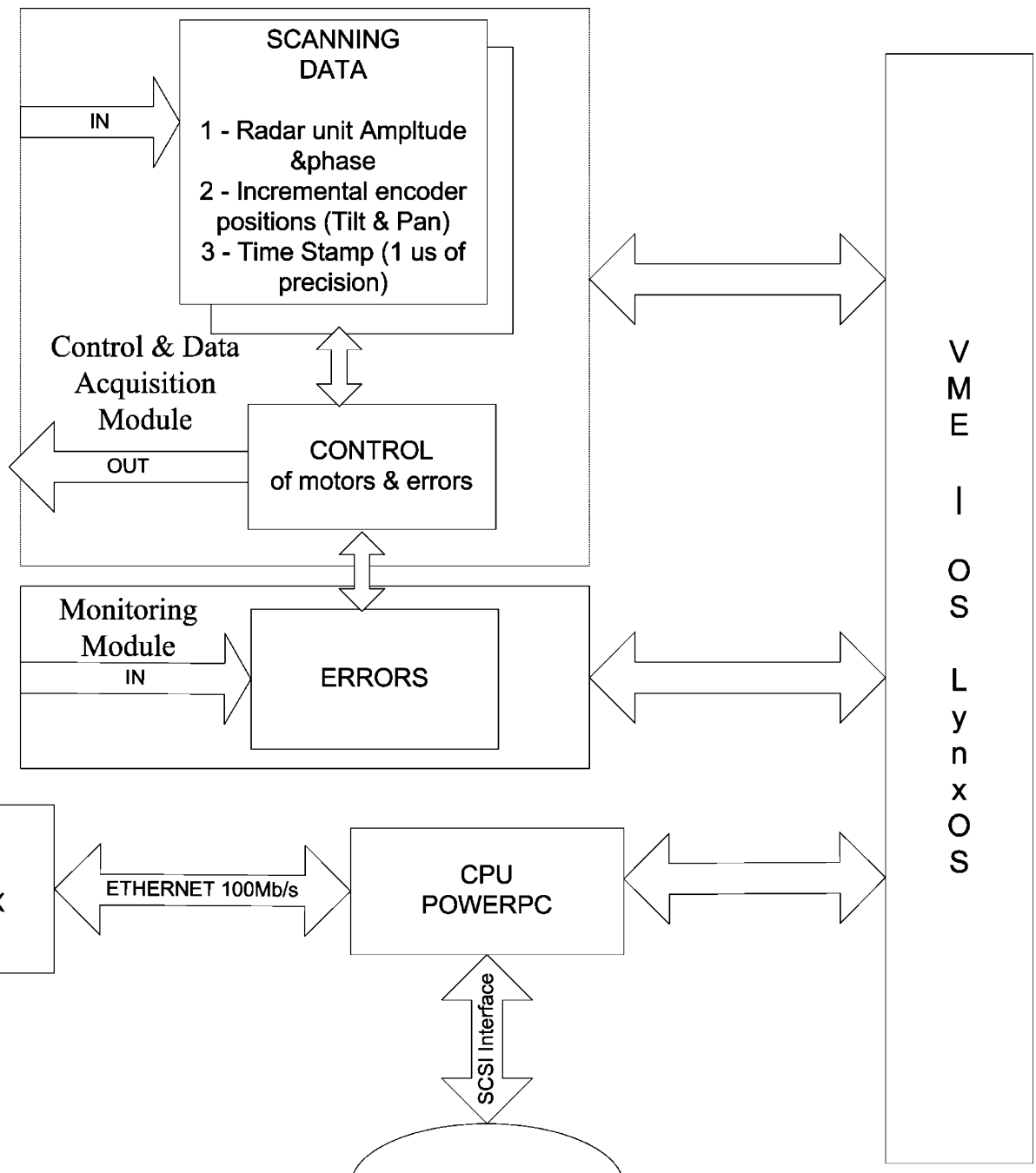

9 GB Hard Disk

Fig. 1. Block diagram of the IVVS. 


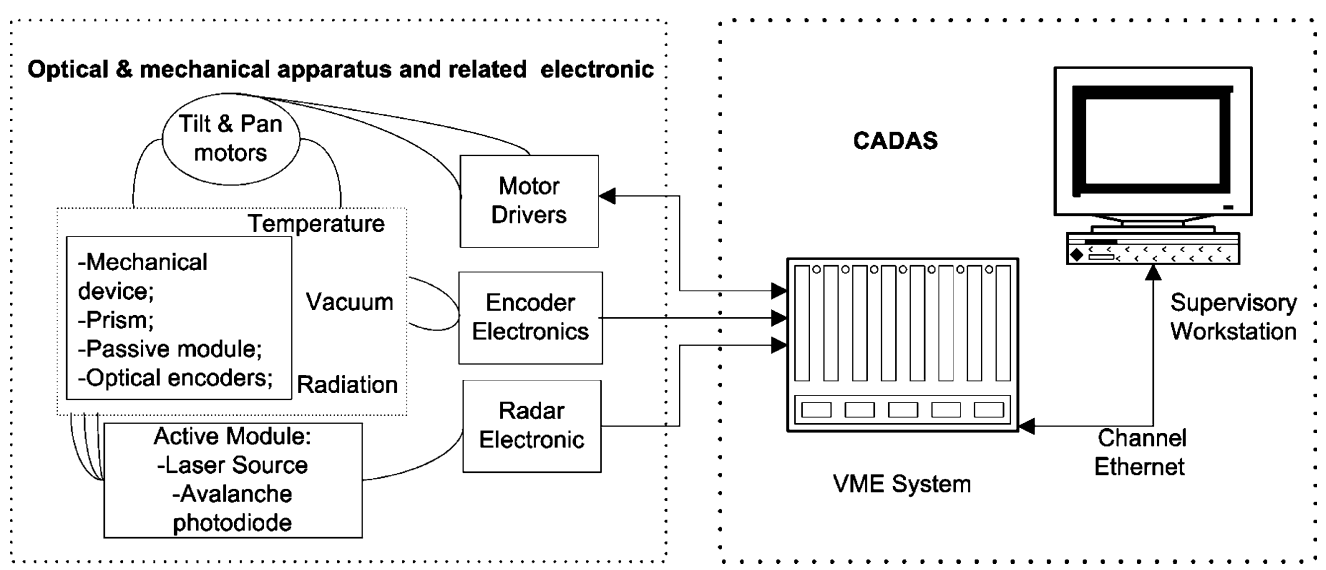

Fig. 2. Block diagram of CADAS.

This control and data acquisition system (CADAS) is an improvement of a similar system that has been developed for the JET laser in-vessel viewing system (LIVVS) [3]. The main upgrades and new features of this system are: (i) the MC68060 microprocessor has been changed to a PowerPC604R-based CPU, in order to guarantee the sustained network data transfer rates of 2 Mbytes/s for $100 \mathrm{kHz}$ pixel clock; (ii) the OS9 operating system has been substituted by LynxOS, aiming at improving the network performance and real-time demands; (iii) the three modules used in the previous system have been reduced to two, leading to a more reliable system and reducing the assembling, test and debug times; (iv) programmable motor speed depending on angular position of the prism has been foreseen, in order to increase the accuracy in the zone of interest and to minimise the overall acquisition time [4]; (v) acquisition of amplitude and phase analogue information from the radar electronic unit has been foreseen, aiming at having redundant information.

This paper is organised as follows: Section 2 contains an overview of the CADAS configuration and provides a detailed description of the two VME modules; the control software is described in Section 3, while the test results as well as some final considerations are presented in Section 4.

\section{System configuration}

The IVVS CADAS is composed of a supervisory workstation connected by a $100 \mathrm{Mbit} / \mathrm{s}$ Ethernet channel to a 12-slot backplane (P1, P2) VME crate. This system houses a PowerPC604R CPU module, with 64 Mbyte RAM, 9 Gbyte hard disk and the LynxOS operating system, as well as two on-site developed modules:

- The intelligent control and data acquisition (CADA) module, which controls the operation of the in-vessel scanning laser and acquires and stores the in-vessel high-resolution image;

- The intelligent monitoring module, which monitors external variables and signals predefined error events.

Fig. 2 depicts a block diagram of the VME system.

The LynxOS operating system has been chosen since it is very suitable for time-critical applications where predictable real-time response is required.

Three levels of software have been developed and implemented for the CADAS operation:

- The low-level software layer, running in the TMS320C31 Digital Signal Processor (DSP) of the two on-site developed modules;

- The middle-level software (TCP/IP Server Application), running on a PPC $400 \mathrm{MHz}$ mi- 
croprocessor on the PowerPC board under LynxOS. Due to its UNIX compatibility and POSIX compliance, migration of applications to and from this operating system (portability) is easily achieved;

- The high-level software (TCP/IP Client Application), running in a Unix Workstation.

The software for these three platforms has been implemented mainly in $\mathrm{C} / \mathrm{C}++$ exploiting its easy use, universality and portability. In some cases DSP assembler has been used just for performance. A software library has also been developed to facilitate future developments and integration in new Workstations.

\subsection{Control and data acquisition module}

Fig. 3 shows a block diagram of the control and data acquisition module. This intelligent module performs the following tasks: (i) control of the scanning motors; (ii) storage of the scanning pixel information in five 32-bit word packet; and (iii) monitoring of the interlocking system state and, in the case of an alarm, stopping the motors and stopping/resetting the running data acquisition process.

Two stepping motors move the prism around two orthogonal axes providing the so-called tilt and pan scans. In order to increase the accuracy in the zone of interest and also to minimise the overall acquisition time, the tilt scanning movement should be carried out at a variable speed depending on the prism angular position according to a previously built lookup table.

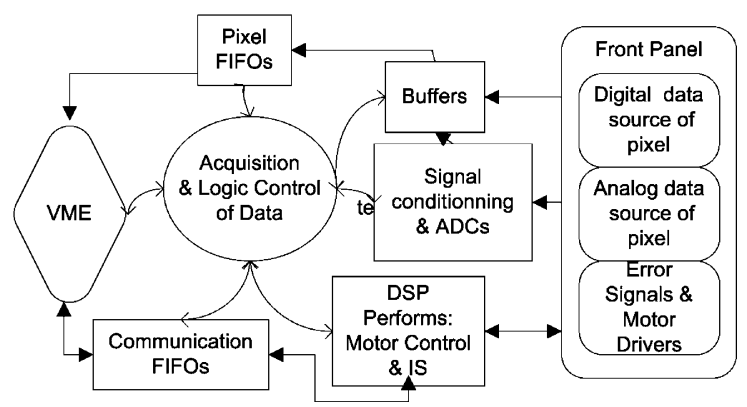

Fig. 3. Block diagram of the control and data acquisition module.
All information enters the board redundantly since it arrives in both digital and analogue forms. This redundancy was needed to sort out problems encountered during off line analysis and it is an efficient security procedure in complex data acquisition systems.

The scanning pixel is composed of three information data types: (i) the backscattered light amplitude and phase information; (ii) the two angular co-ordinates of the scanning prism provided by the encoders, either in analogue or digital form; and (iii) an internal (on-board) time base with a $1 \mu$ s resolution (time stamp). The integrity of this time base is a strong first indicator of no data loss during the process. It also provides means for data checking implementing algorithms.

The interlocking system state is triggered, in real time, by the signals originating from the motor drivers, digital error connector and monitoring modules, aiming at preventing catastrophic situations, as has been already mentioned.

The main features of this module are: (i) two 16-bit ADC channels (LTC1605ACG), with sampling rates up to $100 \mathrm{ksamples} / \mathrm{s}$ and an input range that can be chosen between $\pm 10 \mathrm{~V}$ or \pm 2 $\mathrm{V}$ for the analogue amplitude and phase information; (ii) four, 16-bit ADC channels (ADS7815) with sampling rates up to $250 \mathrm{ksamples} / \mathrm{s}$ and an input range of $\pm 5 \mathrm{~V}$; (iii) two D37 high-density inputs for respectively 24 and 20 TTL signals of the digital amplitude and phase information; (iv) two D25 high-density inputs/outputs for remote control of motors; (v) two D9 inputs for digital encoders; (vi) 1.28 Mbytes of FIFO (First-InFirst-Out) memory for the storage of five 32-bit words; and (vii) local TMS320C31, with 32-bit 512 kwords of memory for data processing.

The logic control of the module is accomplished through complex, in-system programmable logic devices (namely Mach5 and Mach211SP, from Vantis). The in-system programmability feature leads to a higher level of design flexibility and easy upgrading. These programmable devices also integrate the time-stamp 32-bit up counter as well as the tilt and pan up-down 22-bit counters. The digital encoders must give the information of step and direction to activate these two up-down coun- 
ters and a reset TTL signal every time an end-of-turn event of the motor occurs.

\subsection{Monitoring module}

This module is an upgrade of the one used in the JET LIVVS project [3]. It has the following main features: (i) eight 16-bit ADC channels (ADS7815), with a sampling rate of up to 250 ksamples/s and an input range that can vary between $\pm 2.5 \mathrm{~V}$ and $\pm 2.5 \mathrm{mV}$; (ii) up to 64 kbyte FIFO memory per channel; and (iii) local DSP (TMS320C31), with 32-bit 512 kwords of memory for data processing.

The DSP reads the ADC information through the corresponding FIFOs. When a value is outside limits it will force an interrupt in the CADA module that will take the necessary actions.

This module monitors several external variables such as the motor temperature and the probe inclination; it also signals the occurrence of predefined error situations. Up to eight modules can be installed in the same VME crate. The P2 connector performs the interface between the modules installed in the same crate. When more than one monitoring module is needed, the CADA module is able to decide which one must be read using a specially developed 3-bit addressing mode (sent via P2).

\section{Software and communications design structure}

The three software levels communicate amongst themselves in two different ways: (i) the Workstation changes information with the VME crate through the standard TCP-IP protocol over an Ethernet connection (Internet can also be used if the bandwidth is enough); and (ii) the VME main board controls the DSPs through the FIFOs using a dedicated communication protocol.

The TCP-IP communication software is based on a client/server architecture and is divided in two programs: the server and the client applications, which run respectively in the VME crate and the Workstation. The server program acts as a bridge between the VME modules and the client application.
The communication between the client program and the DSPs is also based on a client/server architecture. The DSPs act as servers and are always ready to execute commands sent through the FIFOs.

\subsection{Low-level software}

The low-level software consists of two programs, one running in the control and data acquisition module and the other in the monitoring modules.

The main purpose of the CADA low-level program is to control the stepping-motors. In each acquisition, the motors are programmed with two frequencies. To determine the exact moment to increase or decrease the frequency, the DSP must read the angular prism position from the digital encoders. To avoid motor blocking, acceleration and deceleration ramps have been implemented. Another important purpose of this DSP is to manage the error events. To avoid hardware damage when an error occurs during the execution of a command, the DSP is interrupted, and the necessary actions are immediately taken through the Interrupt Service Routine.

The aim of the monitoring low-level program is to acquire analogue error variables and compare the read data with the safe parameters. If those values are outside the safe parameters, this module interrupts the DSP of the CADA module that manages the errors.

\subsection{Middle-level software}

The main function of the server application (middle-level) software is to acquire data and to act as a bridge between the workstation operator and the VME modules. Each acquisition starts with the initialisation functions that grant process priority, set the acquisition mode (digital or analogue), perform file pre-allocation and management, reset the counters and FIFOs and program the software interrupt. File opening and allocation before the beginning of the data acquisition process are important actions that improve the system performance.

A complete in-vessel inspection represents the 
Table 1

Structure of the IVVS file

\begin{tabular}{llr}
\hline & Description & Size (bytes) \\
\hline Header & File name & 20 \\
& File description & 20 \\
& Ordinal number of the file & 4 \\
& Length in bytes of data packet ${ }^{\mathrm{a}}$ & 4 \\
& Pan Angle begin of the all & 4 \\
& acquisition & \\
& Pan Angle end of the all & 4 \\
& acquisition & \\
& Tilt speed defined by user & 4 \\
& Pan speed defined by user & 4 \\
& First Data packet of current file & 20 \\
& Last Data packet of current file & 20 \\
& Data to be defined & 24 \\
& Fixed long word that sign begin & 4 \\
of Data Pack & \\
The Data Pack & \\
Fixed long word that sign end & 4 \\
of Data Pack & \\
Tata & Xor of all long word in the file \\
(except itself) - Checksum & 4 \\
&
\end{tabular}

a The size of the data packet is variable, but is defined in the header in the item Length in bytes of data packet.

${ }^{\mathrm{b}}$ Each Pixel Information of the Data Pack is already described.

storage of 5 to 9 Gbyte information. In order to easily place a single file in the right position within the set of pre-allocated files, a file structure has been established, once more with some redundant information aiming at simplifying the debugging of the acquired information. Also, for fast debugging, a .cnf text file is generated for each acquired file with the following structure: [Filename] [Number of first pixel in file] [Number of last pixel in file] [First pixel information of file in text mode] [Last pixel information of file in text mode]. Each pixel information has five 32-bit words read in the following order: 1st word - tilt encoder value; 2 nd word - pan encoder value; 3 rd word - amplitude value; 4th word - phase value; 5th word-time stamp. Apart from the time stamp information, which has 32 bits, all the other words have less than 32 bits but more than 16 bits of information. Therefore each information should be read as a 32 bits word. Moreover, data compression is to be implemented. The IVVS file structure is presented in Table 1.

\subsection{High-level software}

The main function of the software running in the Workstation (high-level software) is to control all the low level modules with a suitable and user-friendly graphic interface GUI, to store all acquired data, as well as to reconstruct and visualise the image.

The Unix client program is the most visible part of this fully integrated system. It is the program that the system operator runs to see and analyse the data acquired by the VME system. All the commands such as start/stop acquisition, reset board, definition of acquisition parameters and control of motors, are available from this program. This application was developed to run in a Unix Workstation, but a similar client program may run on other platforms. Since the communication protocol used is standard TCP/IP, available in most operating systems, there should be no difficulty in porting this program to platforms such as Windows NT.

A C Library was developed with all system functionalities to be used as an application program interface.

\section{Test results and final considerations}

CADAS has been tested at CFN-Coimbra Laboratory. Sustainable data transfer rates of about 4 Mbytes/s from the VME system to the Workstation were achieved, although only 2 Mbytes/s from the Workstation to the 18 Gbyte hard disk were necessary.

After these successful tests, CADAS has been installed at the ENEA-Frascati Laboratory, where the other components of the ITER in-vessel vision system (IVVS) have been developed. Operational tests of the IVVS have been performed by complete scans of the interior of the room where the system is installed. Each scan produces a total of 1.2 Gbytes of acquired raw data. The acquisition time necessary to obtain the image was 40 min, at a slow rate of 25 kpixels per second. The image has a resolution of 2372 rows over a $360^{\circ}$ angle and 25 kpixels per row over $180^{\circ}$ (including the dead-zone of the prism) giving 59.3 Mpixels in 


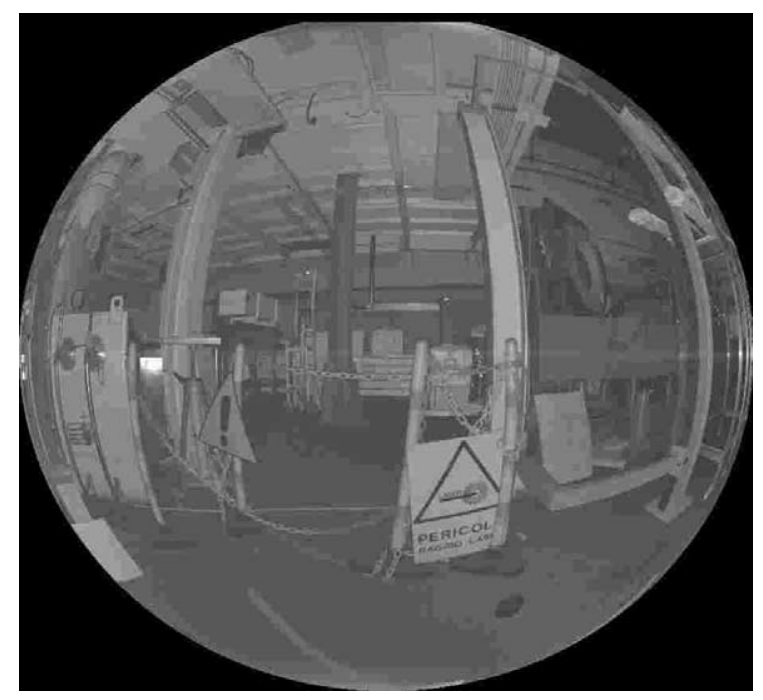

Fig. 4. North view image acquired in a complete scan of the interior of a building.

total. With the $100 \mathrm{kpixels} / \mathrm{s}$ CADAS capability, this image can be taken in $10 \mathrm{~min}$. Data was then processed and divided into north, south, east, west, top and bottom view projection images of the building. Fig. 4 presents the north view image.

These tests confirm that CADAS fulfils the design requirements and is able to guarantee the efficient operation of the IVVS.

The high processing capabilities of the CPU permit real time data compression, which enables pixel clock frequencies above the current $100 \mathrm{kHz}$. Although with the present system requirements this feature is not needed, it is a possibility that could be exploited by future developments.

The application program interface also ensures a good portability to other operating systems and future application developments.

\section{Acknowledgements}

This work has been carried out, in collaboration with ENEA-Frascati, in the frame of the Contract of Association between the European Atomic Energy Community and "Instituto Superior Técnico" and the European Fusion Development Agreement.

\section{References}

[1] L. Bartolini, A. Coletti, M. Ferri de Collibus, G. Fornetti, C. Neri, M. Riva, L. Semeraro, C. Talarico, Amplitudemodulated laser in vessel viewing system (LIVVS) for ITER/JET, in: Proceedings of 20th Symposium on Fusion Technology, September 7-11, 1998, Marseille, France.

[2] A. Coletti, M. Baldarelli, L. Bartolini, A. Bordone, M. Ferri de Collibus, G. Fornetti, S. Lupini, C. Neri, C. Poggi, M. Riva, L. Semeraro, C. Talarico, L. Zannelli, Amplitude-modulated laser viewing system in apparatus for controlled thermonuclear studies, in: American Nuclear Society 8th International Topical Meeting, April 25-29, 1999, Pittsburgh, PA.

[3] R. Pereira, N. Cruz, C. Neri, M. Riva, C. Correia, C.A.F. Varandas, The control and data acquisition system of a laser in-vessel viewing system, Fusion Engineering and Design 48 (1-2) (2000) 205-212.

[4] C. Neri, A. Coletti, ITER Optical Radar Control System Requirement, ENEA Report, July 2000. 